\title{
Growth of continuous bonelike mineral within porous poly(lactide-co-glycolide) scaffolds in vitro
}

\author{
William L. Murphy, ${ }^{1}$ David H. Kohn, ${ }^{1,2}$ David J. Mooney ${ }^{1-3}$ \\ ${ }^{1}$ Department of Biomedical Engineering, University of Michigan, Ann Arbor, Michigan 48109 \\ ${ }^{2}$ Department of Biologic and Materials Sciences, University of Michigan, Ann Arbor, Michigan 48109 \\ ${ }^{3}$ Department of Chemical Engineering, University of Michigan, Ann Arbor, Michigan 48109
}

Received 15 July 1999; revised 2 August 1999; accepted 24 August 1999

\begin{abstract}
Strategies to engineer bone have focused on the use of natural or synthetic degradable materials as scaffolds for cell transplantation or as substrates to guide bone regeneration. The basic requirements of the scaffold material are biocompatibility, degradability, mechanical integrity, and osteoconductivity. A major design problem is satisfying each of these requirements with a single scaffold material. This study addresses this problem by describing an approach to combine the biocompatibility and degradability of a polymer scaffold with the osteoconductivity and mechanical reinforcement of a bonelike mineral film. We report the nucleation and growth of a continuous carbonated apatite mineral on the interior pore surfaces of a porous, degradable polymer scaffold via a one step, room temperature incubation process. A 3-dimensional, porous scaffold of the copolymer 85:15 poly(lactide-co-glycolide) was fabricated by a solvent casting, particulate leaching process. Fourier transform IR spectroscopy and scanning electron microscopy (SEM)
\end{abstract}

analysis after different incubation times in a simulated body fluid (SBF) demonstrate the growth of a continuous bonelike apatite layer within the pores of the polymer scaffold. Quantification of phosphate on the scaffold displays the growth and development of the mineral film over time with an incorporation of $0.43 \mathrm{mg}$ of phosphate (equivalent to $0.76 \mathrm{mg}$ of hydroxyapatite) per scaffold after 14 days in SBF. The compressive moduli of polymer scaffolds increased fivefold with formation of a mineral film after a 16-day incubation time as compared to control scaffolds. In summary, this biomimetic treatment provides a simple, one step, room temperature method for surface functionalization and subsequent mineral nucleation and growth on biodegradable polymer scaffolds for tissue engineering. (c) 2000 John Wiley \& Sons, Inc. J Biomed Mater Res, 50, 50-58, 2000.

Key words: tissue engineering; biomimetic; mineralization; apatite; bone

\section{INTRODUCTION}

Reconstruction of skeletal defects represents a major clinical challenge with over 1 million bone grafting procedures performed each year. ${ }^{1}$ Novel strategies for regenerating diseased or damaged bone tissue are necessary because of limitations in conventional therapies for the treatment of trauma, congenital defects, cancer, and other bone diseases. Supply of autograft tissue is limited, and there is potential for pathogen transfer from an allogeneic graft. A cell-based approach being developed by several investigators involves seeding of autologous cells onto synthetic, degradable materials in vitro prior to implantation. ${ }^{2-4}$ In this approach a porous, 3-dimensional synthetic construct can provide

Correspondence to: Dr. D. J. Mooney; e-mail: mooneyd@ engin.umich.edu or Dr. D. H. Kohn; e-mail: dhKohn@umich. edu

(c) 2000 John Wiley \& Sons, Inc. a substrate for cell growth and proliferation, and cells may eventually secrete extracellular matrix and form new tissue. During the process of new tissue formation in vivo the synthetic construct degrades, leaving a natural tissue replacement. An alternative approach, termed guided tissue regeneration (GTR), is to directly implant the synthetic construct in vivo without cells and allow adjacent cells to migrate onto the surface of the material and form new tissue. ${ }^{5}$ In each of these tissue engineering approaches, the degree of success likely depends on the properties of the synthetic material. Engineering of a bony tissue in vitro or in vivo will likely require a scaffold with specific properties that are beneficial to bone regeneration.

Key factors in the success of a tissue engineering scaffold are biocompatibility, degradability, mechanical integrity, and osteoconductivity. Biocompatibility and degradability can be controlled by the appropriate choice of synthetic material. Poly $(\alpha$-hydroxy acids) such as poly(lactic acid) and poly(glycolic acid), are considered biocompatible and degrade over controllable time scales into natural metabolites. These mate- 
rials are commonly chosen as synthetic scaffold materials for a variety of tissue engineering applications. ${ }^{2,6,7}$

Investigators have attempted to enhance osteoconductivity and in some cases mechanical integrity by coating orthopedic and dental implant materials with a continuous bonelike mineral (BLM) via a room temperature process. ${ }^{8}$ Materials that have been investigated include bioceramics, ${ }^{9-11}$ metals, ${ }^{12,13}$ and polymers, ${ }^{14,15}$ bioceramics being the most active area of study. Although differing in their specific processing techniques, all strategies for growth of a BLM layer include three basic steps: surface functionalization to create a negatively charged surface, surface calcification via chelation of calcium ions, and subsequent growth of the BLM layer. Surfaces have been functionalized by such techniques as binding of hydrated silicate ions to a bioceramic, ${ }^{9-11}$ utilization of selfassembling monolayers, ${ }^{12}$ and covalent grafting of molecules to polymers. ${ }^{14}$ Surface calcification is generally accomplished by increasing the local calcium concentration near the surface of the implant via an ion exchange mechanism between a calcium rich solution and a calcium containing bioceramic. In all cases the mineral is subsequently grown by incubation in a simulated body fluid (SBF) that contains the ionic constituents of blood plasma.

The osteoconductivity and mechanical integrity of a tissue engineering scaffold can also potentially be enhanced by the growth of a BLM on the surface of the synthetic construct prior to cell seeding. The presence of a surface BLM layer has been shown to be a prerequisite to conduction of osteogenic cells into various porous synthetic scaffolds ${ }^{16,17}$ or onto the surfaces of bioactive glasses. ${ }^{18}$ The BLM layer has been associated with increased bioreactivity, ${ }^{19}$ and it may be responsible for the bonding of bioactive ceramics to preexisting bone. ${ }^{20,21}$ Until recently there has been little work done on mineralization of degradable materials, ${ }^{22-24}$ and there has been no demonstration of a continuous BLM within the pores of a 3-dimensional, porous, degradable scaffold. The growth of a continuous BLM layer could also enhance the mechanical integrity of a synthetic construct via a reinforcement mechanism. Polymer constructs used for tissue engineering applications are generally highly porous and do not have mechanical properties in the same range as bone. ${ }^{6,25}$ An interconnected mineral coating over the inner pore surfaces of a polymer construct may provide a hard and stiff exoskeleton, increasing its modulus and enhancing its resistance to cellular contractile forces during tissue development.

This article presents a strategy for the nucleation and growth of a continuous BLM layer on the inner pore surfaces of 3-dimensional, porous scaffolds of the biodegradable copolymer poly(lactide-co-glycolide) (PLG). PLG was a convenient choice because it is a linear polyester, and therefore surface functionalization can be readily achieved via a simple hydrolysis reaction. The hydrolysis reaction can occur in an aqueous mineral growth solution, allowing for a single step mineralization process. This single step, room temperature process is shown to be adequate for functionalization, mineral nucleation, and growth of a continuous BLM layer within the pores of a 3-dimensional, porous PLG scaffold.

\section{MATERIALS AND METHODS}

\section{Sample preparation}

PLG pellets with a lactide/glycolide ratio of 85:15 were obtained from Medisorb, Inc. (inherent viscosity, IV, $=0.78$ $\mathrm{dL} / \mathrm{g}$ ) and Boehringer-Ingelheim Inc. (IV $=1.5 \mathrm{dL} / \mathrm{g}$ ). Higher IV PLG was used in studies measuring mechanical properties due to the greater initial mechanical integrity of high IV PLG scaffolds. Scaffolds were prepared via a solvent casting, particulate leaching process described elsewhere. ${ }^{26}$ The scaffolds were circular disks with a diameter of $12 \mathrm{~mm}$ and a thickness of $3 \mathrm{~mm}$. The pore size range was controlled by using $\mathrm{NaCl}$ particles with a diameter of $250-450 \mu \mathrm{m}$ in the processing. The porosity of scaffolds was calculated before and after mineral film formation using the known density of the solid polymer, the known density of carbonated apatite ${ }^{27}$ the measured mass of mineral and polymer in the scaffold, and the measured volume of the scaffold.

Scaffolds were each incubated in a $50-\mathrm{mL}$ solution of SBF for periods from 0 to 16 days for mineral film formation. The SBF solution was changed every $24 \mathrm{~h}$ to ensure sufficient ion concentrations for mineral growth. The SBF was prepared by dissolving the following reagents in deionized water: 141 $\mathrm{mM} \mathrm{NaCl}, 4.0 \mathrm{mM} \mathrm{KCl}, 0.5 \mathrm{mM} \mathrm{MgSO}_{4}, 1.0 \mathrm{mM} \mathrm{MgCl}_{2}, 4.2$

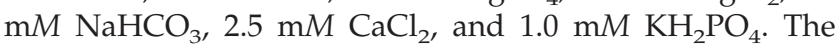
resulting SBF was buffered to $\mathrm{pH} 7.4$ with Tris- $\mathrm{HCl}$ and was held at $37^{\circ} \mathrm{C}$ for the duration of the incubation period. Control samples were incubated in $50 \mathrm{~mL}$ of Tris- $\mathrm{HCl}$ solution buffered to $\mathrm{pH} 7.4$ and held at $37^{\circ} \mathrm{C}$ for the duration of the incubation period. Before and after SBF incubation each scaffold was lyophilized prior to analysis.

\section{Materials characterization}

\section{Electron microscopy}

Scaffolds were bisected after various incubation times via freeze fracture. Following bisection, scaffolds were sputter coated with a film of gold-palladium alloy (AuPd) and imaged under a high vacuum. Images were obtained using a Hitachi S-800 field emission SE microscope operating at $5 \mathrm{kV}$. 


\section{IR spectroscopy}

Fourier transform IR spectroscopy (FTIR) data were obtained using an Avatar 360 FTIR ESP spectrometer from Nicolet Instrument Corp. Scaffolds were ground into a fine powder and pressed into a $\mathrm{KBr}$ pellet. Spectra were obtained following 0-, 2-, 6-, 10-, and 16-day incubation times and compared to reveal changes in scaffold composition with increasing SBF incubation time.

\section{Mass measurements}

The mass of each scaffold was measured before and after incubation in SBF. The percent increases in mass were calculated and compared with mass changes of control scaffolds. The percent changes in the mass of experimental scaffolds were compared using analysis of variance (ANOVA) to reveal significant differences with SBF incubation time. Significant differences in the percent mass change of experimental scaffolds versus control scaffolds at each incubation time point were analyzed using a Student's $t$ test.

\section{Phosphate assays}

The amount of phosphate present in the scaffolds incubated for $0,4,8,12$, and 14 days was determined using a variation of a previously described assay. ${ }^{28}$ In brief, following SBF incubation the scaffolds were dissolved in $66 \%$ (11.54M) acetic acid solution. A 3.4-mL aliquot of the dissolved scaffold solution was added to $1.6 \mathrm{~mL}$ of $125 \mathrm{mM}$ ammonium molybdate solution. The amount of phosphate was quantitatively detected by measuring the absorbance of the 400-nm band on a UV-visible (UV-vis) spectrophotometer and comparing it to a set of standards with known phosphate concentrations and equal concentrations of acetic acid and ammonium molybdate. Standards for the quantitative determination of phosphate content had a correlation coefficient greater than or equal to 0.999 for the concentration range of $60-300 \mu \mathrm{M}$. The $400-\mathrm{nm}$ absorption spectra were collected using a Perkin Elmer lambda 12 UV-vis spectrophotometer. To estimate the amount of apatite on the scaffold after the 14-day incubation, the measured mass of phosphate was multiplied by the known ratio of the mass of hydroxyapatite $\left[\mathrm{Ca}_{10}\left(\mathrm{PO}_{4}\right)_{6}(\mathrm{OH})_{2}\right.$, formula weight $=1004.36$ $\mathrm{g}]$ to the mass of phosphate in hydroxyapatite (569.58 g). This is a conservative estimate, because it assumes that all phosphate is being incorporated into stoichiometric hydroxyapatite. This mineral mass estimate would increase with increasing substitution of carbonate in the mineral crystal. The values for phosphate mass over time were compared using ANOVA.

\section{Measurement of moduli}

The compressive moduli of scaffolds before and after SBF incubation were determined using an MTS Bionix 100 mechanical testing machine. Samples were compressed between platens with a constant deformation rate of $1 \mathrm{~mm} /$ min. The compression plates had a diameter of $45 \mathrm{~mm}$ and thus covered the entire 12-mm diameter surface of the scaffold. A small preload was applied to each sample to ensure that the entire scaffold surface was in contact with the compression plates prior to testing, and the distance between plates prior to each test was equal to the measured thickness of the scaffold being tested. Compressive moduli were determined for all scaffolds before incubation and for each of three samples for each incubation time. At each time point, experimental moduli were compared to control moduli via a Student's $t$ test to determine significant differences in compressive modulus. The moduli of experimental scaffolds were compared using ANOVA to reveal significant differences in compressive modulus with SBF incubation time.

\section{Statistical analysis}

Three scaffolds were prepared, treated, and analyzed for SBF incubation times of 0-16 days. The values on our graphs represent means and standard deviations. Statistical analysis was done using InStat software, version 2.01.

\section{RESULTS}

Incubation of 85:15 PLG scaffolds in SBF led to the nucleation and subsequent growth of a continuous carbonated apatite film on the inner surfaces of pores. Cross-sectional micrographs of the inner pore surfaces of treated scaffolds show that the initially smooth pore surface [Fig. 1(a)] begins to be coated by small mineral crystals by 6 days [Fig. 1(b)], which grow over time [Fig. 1(c)] into a continuous film [Fig. 1(d)]. Overall, the majority of the inner pore structure of the scaffold treated for 16 days was covered by a continuous layer, and there was no evidence of disperse mineral crystals in the scaffolds. Micrographs of the cross section of the 16-day incubated scaffolds at three different magnifications show the large scale pore structure of the scaffold and the cross section of the continuous mineral coating on each side of a pore wall (Fig. 2).

The FTIR analysis was performed to gain information about the composition of the mineral formed on the inner pore surfaces of the scaffold. FTIR spectra of scaffolds treated for $0,2,6,10$, and 16 days indicate the growth of a carbonated apatite mineral within the scaffold (Fig. 3). The peaks at 461, 596, 956, and 1036 $\mathrm{cm}^{-1}$ are indicative of the $v_{2}, v_{4}, v_{1}$, and $v_{3}$ vibrations of $\mathrm{PO}_{4}^{3-}$, respectively. ${ }^{29,30}$ The peaks at 1552 and 1630 $\mathrm{cm}^{-1}$ are indicative of the $\nu_{3}$ vibration of carbonate, and the peak at $866 \mathrm{~cm}^{-1}$ is indicative of carbonate $\nu_{2}$. Each of these peaks increases with incubation time, representing the growth of a carbonated apatite mineral. Relative peak intensities are in accordance with results from previous FTIR studies of carbonated apatites $^{29}$ with the exception of the carbonate peaks. The 


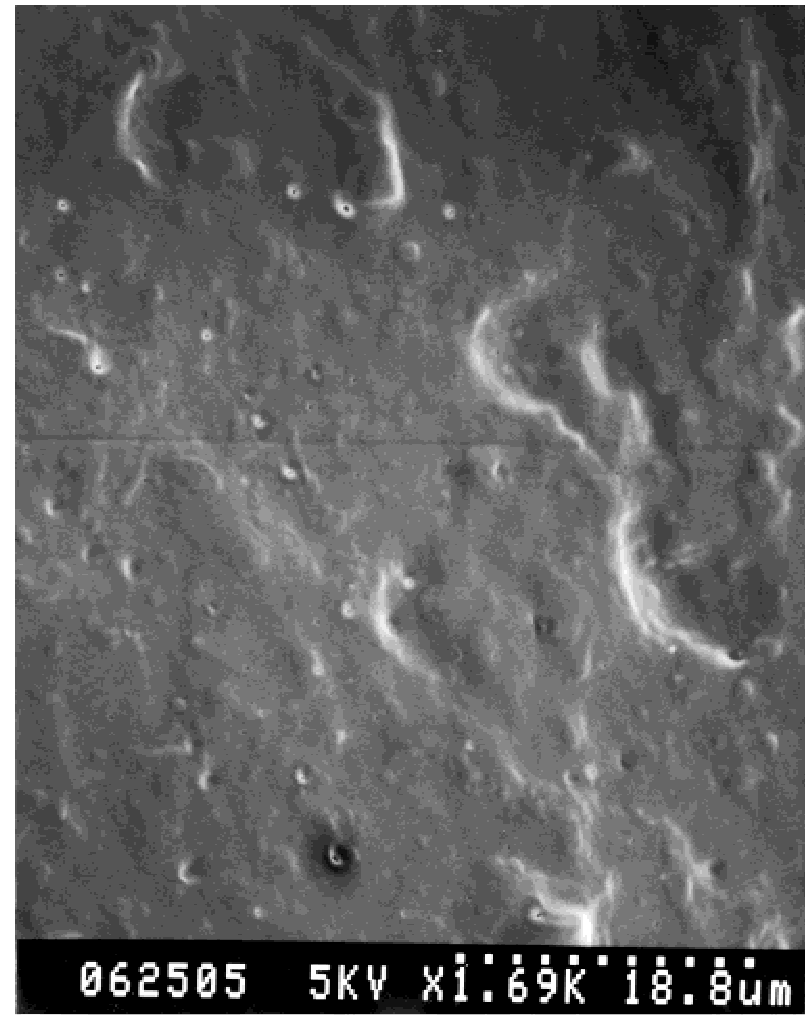

(a)

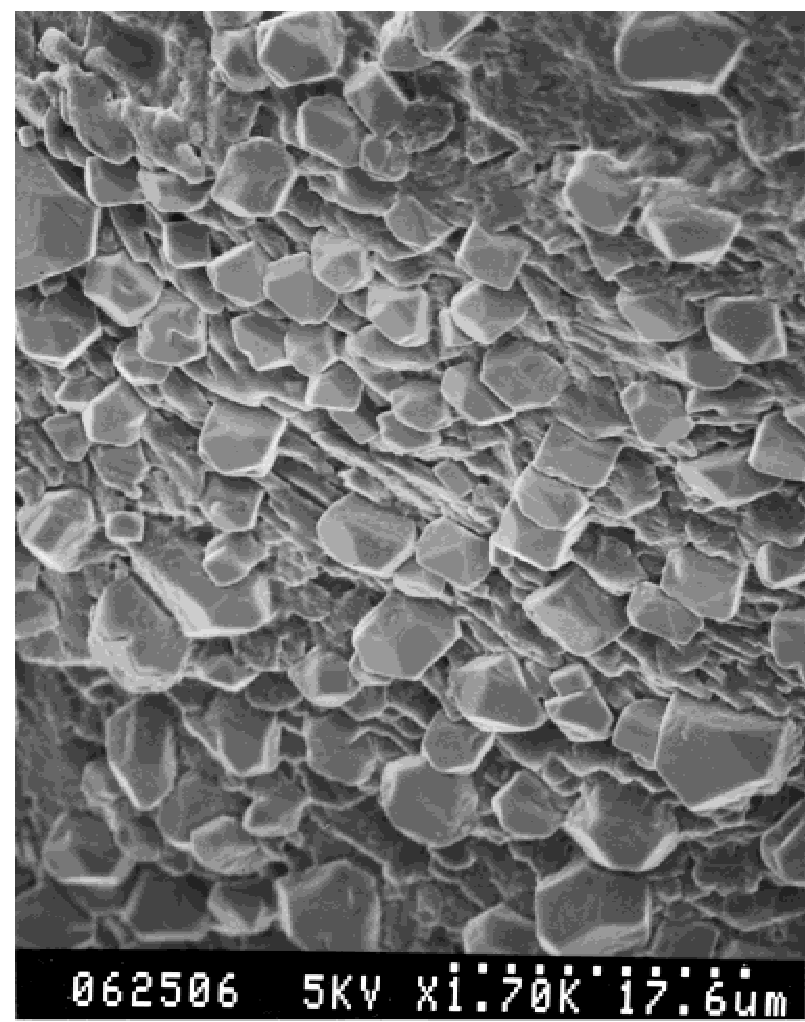

(c)

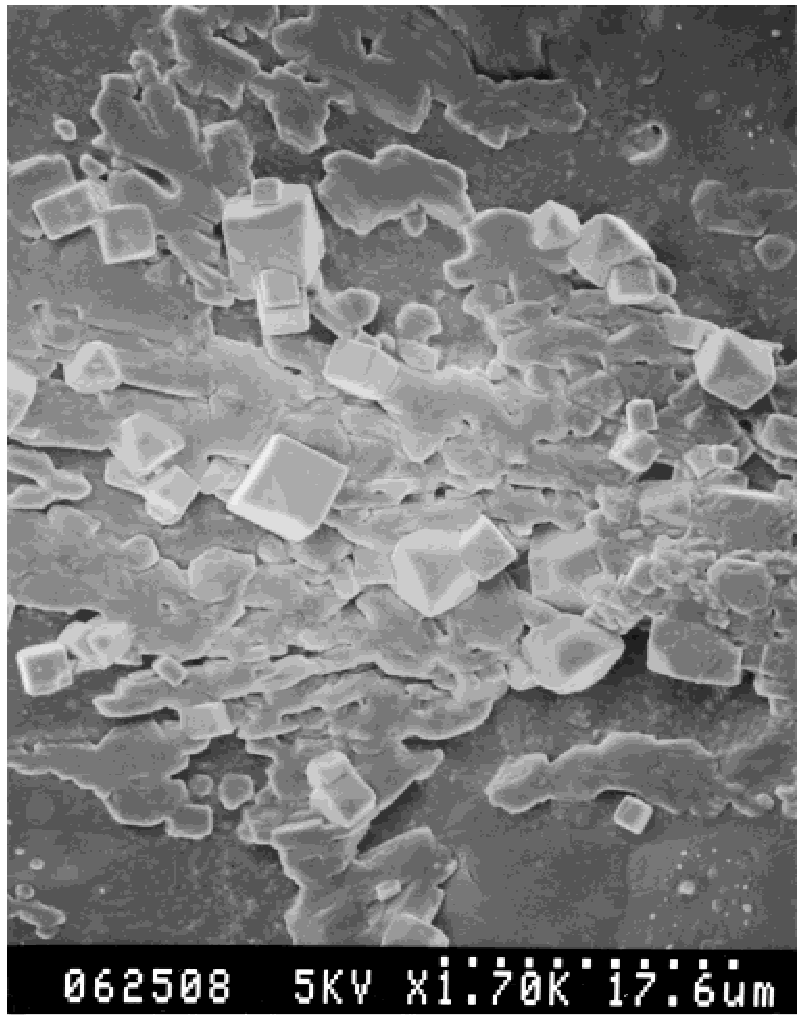

(b)

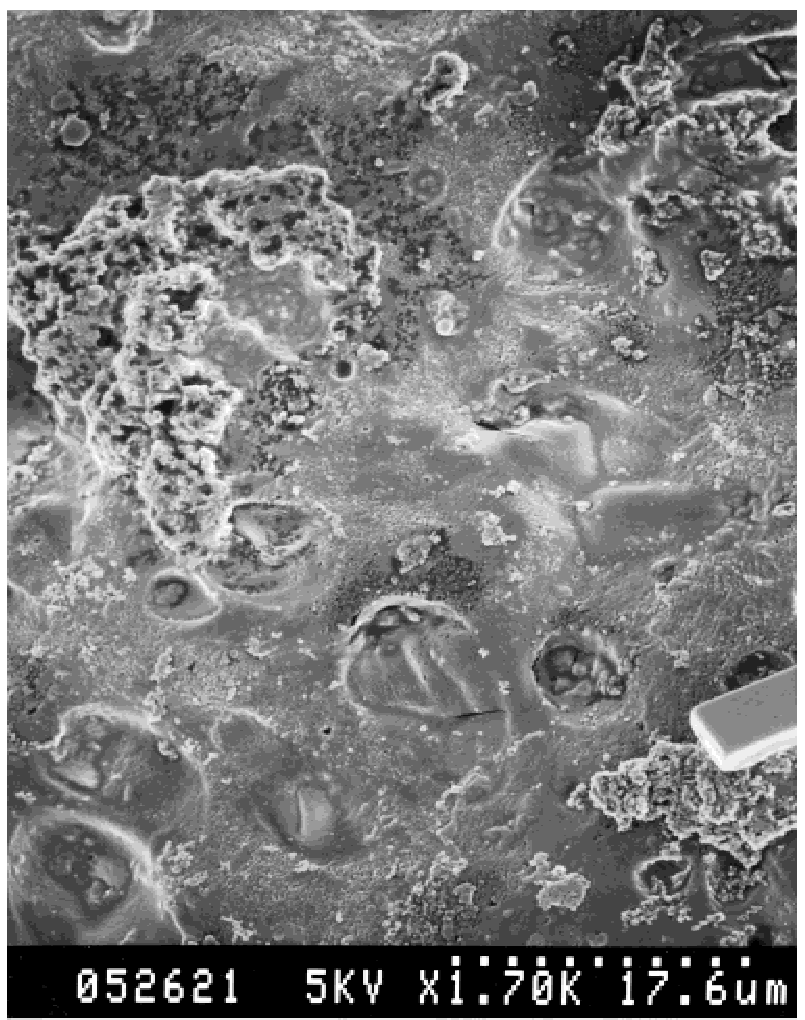

(d)

Figure 1. SEM micrographs (original magnification X1700) of 85:15 PLG scaffolds. Shown are the inner surface of a single pore of (a) the control scaffold prepared by a solvent casting particulate leaching process and not incubated in SBF, (b) scaffold incubated in SBF for 6 days displaying small mineral crystals growing on the polymer substrate, (c) the scaffold incubated in SBF for 10 days displaying a large density of mineral crystals growing on the polymer substrate, and (d) the scaffold incubated in SBF for 16 days displaying a continuous mineral film grown on the polymer substrate. 


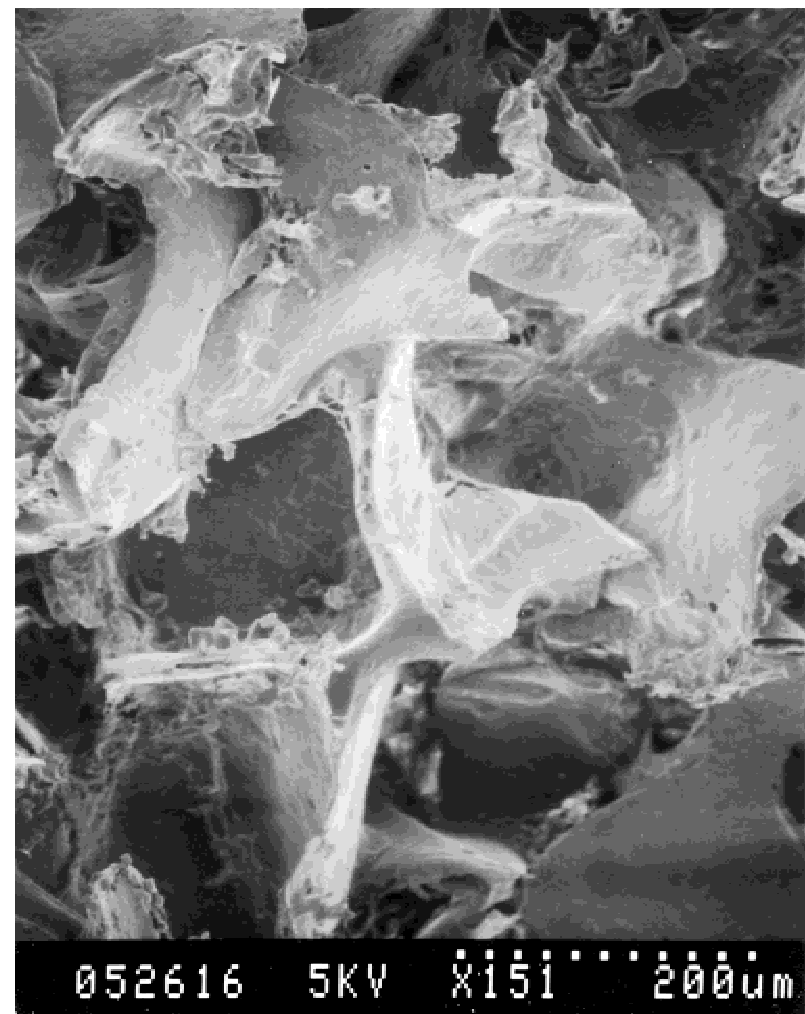

(a)

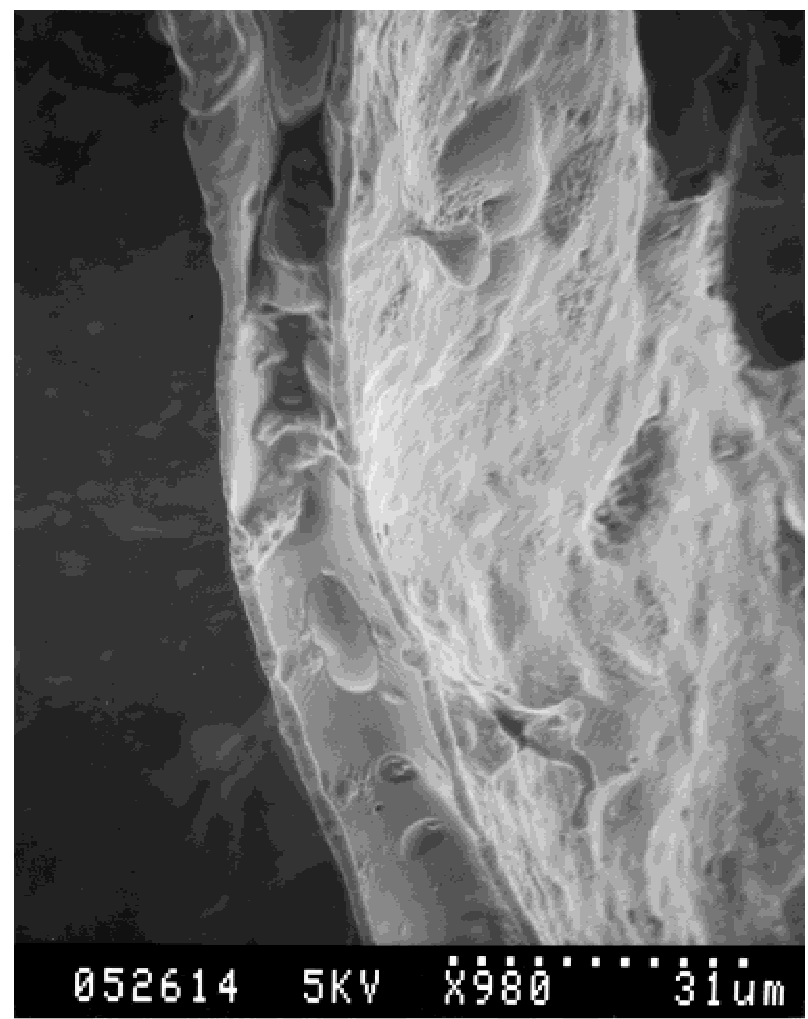

(c)

carbonate peaks have a larger relative intensity than expected, most likely due to the contribution from carboxylate anions present in the polymer scaffold. The peaks at 1755 and $1455 \mathrm{~cm}^{-1}$ represent PLG acid

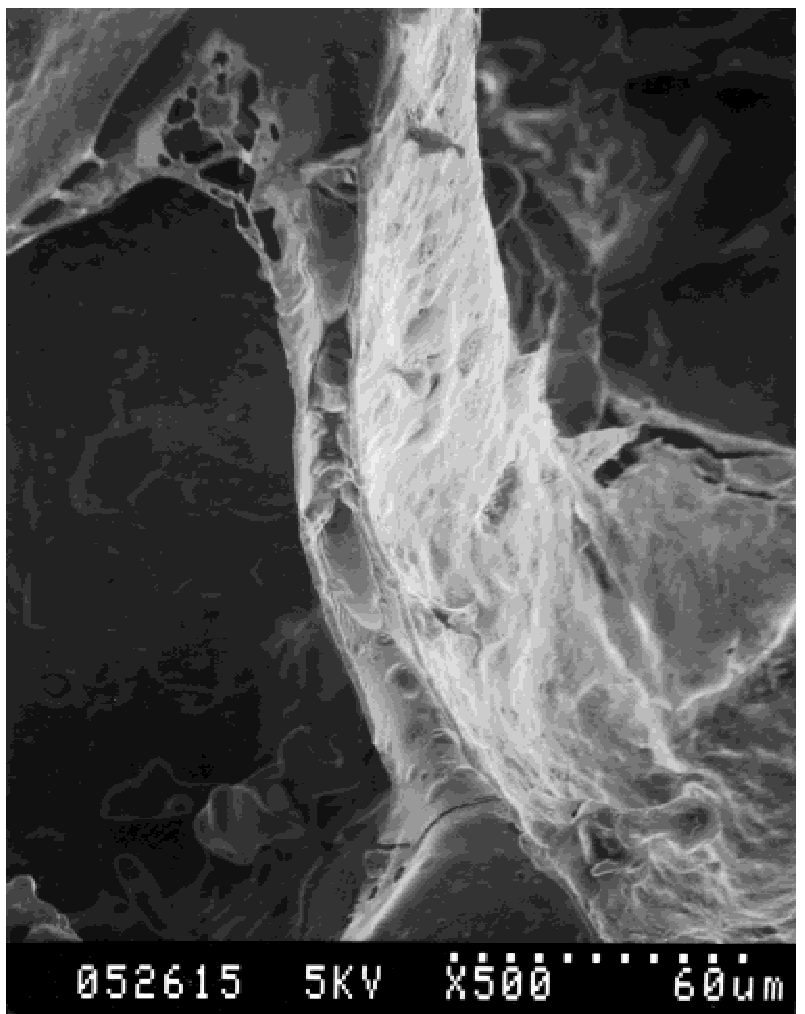

(b)

Figure 2. SEM micrographs of the cross section of an 85:15 PLG scaffold incubated in SBF for 16 days. These micrographs at three different magnifications show (a) the large scale pore structure of the scaffold and focus on $(b, c)$ the cross section of a mineralized pore wall at higher magnifications.

(PLGA), and the peak at $1091 \mathrm{~cm}^{-1}$ is indicative of C-O stretch in the ester.

The scaffolds demonstrated an increase in mass over time, culminating in a $11 \pm 2 \%$ mass gain at the 


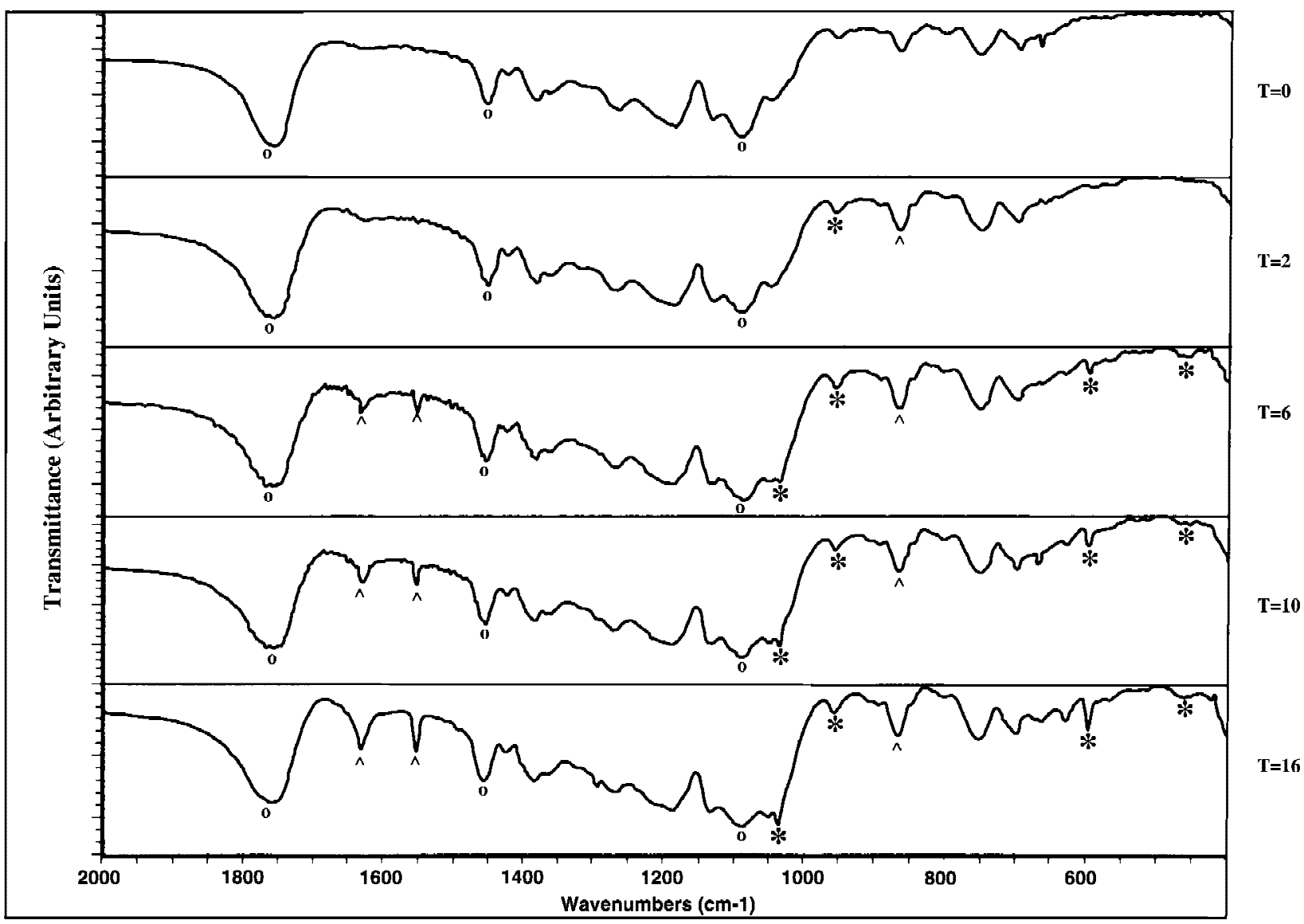

Figure 3. FTIR spectra displaying development of $(*)$ phosphate and $(\wedge)$ carbonate peaks with increasing incubation time in SBF. (o) The peaks representing poly(lactide-co-glycolide) are also labeled. Incubation times are given to the right of each spectrum.

end of the 16-day incubation (Fig. 4). ANOVA of the percent mass changes of experimental scaffolds reveal a significant difference in scaffold mass over time $(p<$ $0.05)$, while ANOVA of the percent mass changes of

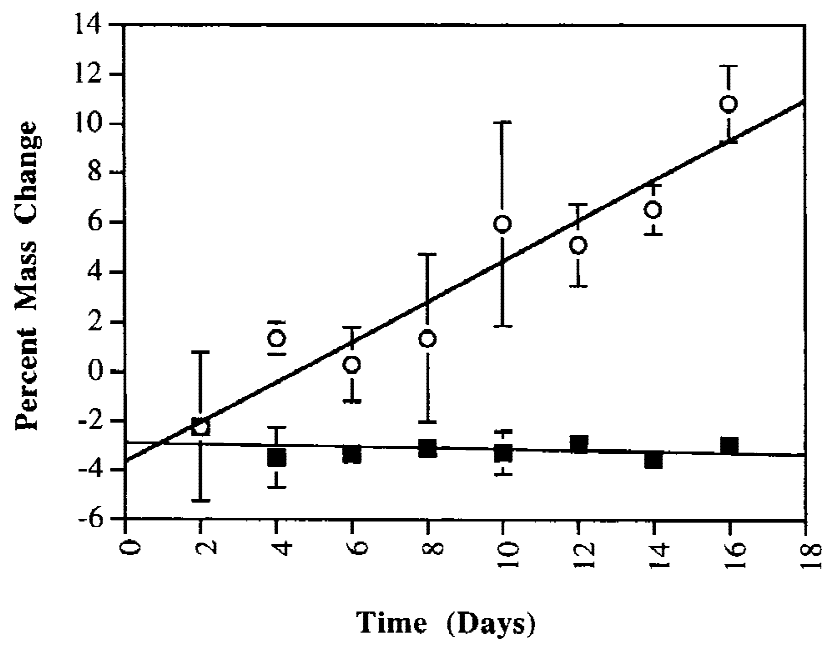

Figure 4. The percent mass increase vs. incubation time of experimental scaffolds incubated in (o) SBF, and ( $\square$ ) control samples incubated in Tris buffer ( $\mathrm{pH}$ 7.4). The values represent the means and standard deviations $(n=3)$. control scaffolds does not show a significant difference over time $(p>0.05)$. The percent mass changes of experimental samples and control samples were significantly different for each time point beyond 8 days $(p<0.05)$. To confirm that the increase in mass was caused by deposition of an apatitic mineral, the mass of phosphate in the scaffolds was analyzed next. Phosphate content within the treated scaffolds also increased significantly with the treatment time (Fig. 5). A comparison of phosphate masses via ANOVA showed a statistically significant increase over time $(p$ $<0.05)$, and the differences in phosphate mass between days 8 and $12(p<0.05)$ and days 12 and $14(p$ $=0.05)$ were also statistically significant. If one assumes that all phosphate is present as pure hydroxyapatite, the $0.43 \mathrm{mg}$ phosphate mass is equivalent to $0.76 \mathrm{mg}$ of hydroxyapatite per scaffold. The measured overall mass increase of the scaffold (Fig. 4) was $1.02 \pm$ $0.40 \mathrm{mg}$ at 14 days. The fact that the overall mass increase was larger than the estimated hydroxyapatite value suggested carbonate substitution in the mineral crystal.

Growth of the BLM layer significantly increased the compressive modulus of 85:15 PLG scaffolds (Fig. 6) without a significant decrease in scaffold porosity. The 


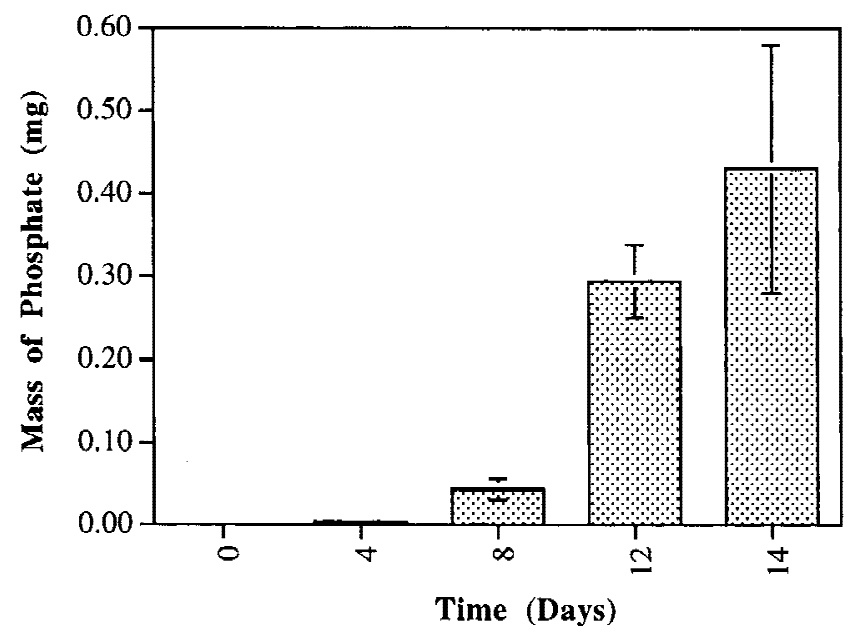

Figure 5. The mass of phosphate present in the scaffolds vs. incubation time. The values represent the means and standard deviations $(n=3)$.

compressive modulus increased from $60 \pm 20 \mathrm{kPa}$ before treatment to $320 \pm 60 \mathrm{kPa}$ after a 16 -day treatment, a fivefold increase in modulus. ANOVA of modulus changes of experimental scaffolds revealed a significant difference in scaffold modulus over time $(p<$ 0.05), while ANOVA of control modulus data did not show a significant difference over time $(p>0.05)$. The differences between the moduli of experimental scaffolds and the moduli of control scaffolds were statistically significant for treatment times of 10 days or longer $(p<0.05)$. The porosity of the scaffolds did not decrease appreciably after incubation in SBF. Untreated scaffolds were $95.6 \pm 0.2 \%$ porous, while scaffolds incubated in SBF for 16 days were $94.0 \pm 0.30 \%$ porous $(n=3)$. This agrees with the electron micrographs (Fig. 2), which display only a thin (1-10 $\mu \mathrm{m})$ mineral coating and thus no significant change in pore size due to mineral growth.

\section{DISCUSSION}

A biomimetic, single step, room temperature process was used to nucleate and grow a continuous BLM on the inner pore surface of a 3-dimensional, porous, degradable polymer construct. A 16-day treatment was adequate for growth of a continuous layer on much of the inner pore surface. Growth of this continuous mineral film significantly increased the compressive modulus of the highly porous construct without a large decrease in total porosity. The mineral grown was a carbonated apatite mineral, similar to the mineral present in tooth and bone tissue, and hence is expected to have a high degree of bioreactivity.

The process of mineral growth on these scaffolds occurs in three phases. The PLG surface is hydrolyzed

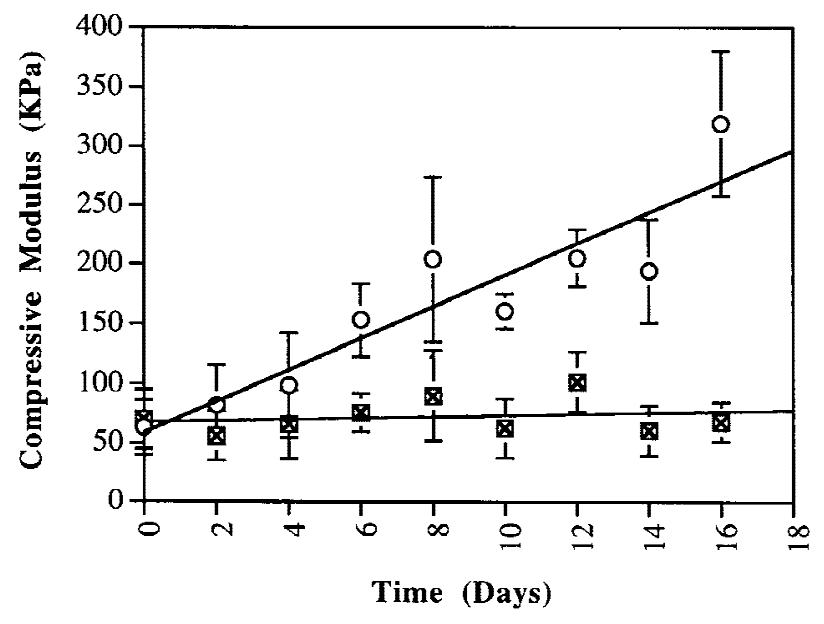

Figure 6. The compressive modulus vs. incubation time for experimental scaffolds incubated in (o) SBF, and (区) control scaffolds incubated in Tris buffer ( $\mathrm{pH} 7.4$ ). The values represent the means and standard deviations $(n=3)$.

by water molecules in solution, converting ester bonds in the polymer into surface carboxylic acid groups. ${ }^{31}$ These groups become carboxylate anions at the solution $\mathrm{pH}(7.4)$ and therefore provide a negatively charged substrate surface. The negatively charged surface chelates calcium ions in solution. The binding of these soluble ionic precursors to mineral formation stimulates surface nucleation, and mineral growth follows.

The process of surface functionalization and subsequent mineral growth is a biomimetic process analogous to several natural processes, including the process of mineral formation in teeth and bones and the process of shell development on mollusks. Proteins present in the ground substance of tooth and bone tissues contain phosphate groups that are negatively charged at physiological $\mathrm{pH} .{ }^{32}$ Carboxylic acid groups on the aspartic acid containing proteins in mollusks are also negatively charged in the mollusk environment, much like the surface carboxylic acid groups in this study. ${ }^{33}$ Although the role of the negatively charged groups in nucleating mineral is poorly understood, the process can be readily mimicked to produce calcium phosphate coatings on various surfaces. In this study the process of mineralization in tooth and bone development is directly mimicked through the use of an SBF as the mineral growth solution. The process of mollusk shell development is also directly mimicked through the use of surface carboxylic acid groups to form a continuous mineral coating (or shell) on the polymer construct. The phase, morphology, and constitution of the mineral in this process can be potentially controlled by varying the $\mathrm{pH}$, ionic concentrations, or temperature of the mineral growth solution. ${ }^{34,35}$

Quantification of phosphate content suggests that the majority of mineral growth occurred between days 
8 and 12 (Fig. 5). These data are consistent with SEM micrographs (Fig. 1), which demonstrate a low density of small crystals on the pore surface after a 6-day incubation but high density of larger crystals after a 10day incubation. The increase in phosphate content from day 12 to day 14 likely represents mineral growth into a continuous layer, because SEM micrographs demonstrated a continuous mineral layer after a 16-day incubation. The growth of a continuous mineral layer in this study is in contrast to previous studies of mineral growth on other polyester surfaces, which do not show growth of continuous BLM after incubation in SBF. Incubation of poly(methyl methacrylate), and poly(ethylene terephthalate) in a solution with 1.5 times the ionic concentrations of SBF for 6 days resulted in no nucleation of the mineral. ${ }^{15}$ Incubation of poly(L-lactic acid) (PLLA) scaffolds in SBF for 15 days showed nucleation and growth of many microparticles of mineral but no continuity. ${ }^{36}$ The difference in the results of this study compared to those of previous studies can be explained by the rapid surface hydrolysis of 85:15 PLG relative to previously studied materials. A decrease in the lactide/glycolide ratio of the copolymer would increase the rate of surface hydrolysis; however, the mechanical integrity of these polymers declines with increasing glycolide content. $^{37}$ Thus, although a relatively large amount of glycolide may facilitate mineral formation via rapid surface hydrolysis, there is a trade-off in that this would also result in a decrease in mechanical integrity of the polymeric component of the scaffold. However, the increased strength of the composite due to the mineral layer may offset this effect, and the overall strength of the scaffold may increase.

The scaffold material developed in this study develops a continuous mineral layer and has a posttreatment compressive modulus of $320 \pm 60 \mathrm{kPa}$ after a 16-day treatment. This modulus is larger than that of other poly $(\alpha$-hydroxy acid) materials used for bone tissue engineering, ${ }^{38,39}$ and it is also larger than the moduli of PLLA bonded poly(glycolic acid) (PGA) matrices that have been shown to be adequate for resistance of cellular forces during smooth muscle tissue development. ${ }^{6}$ The significant increase in modulus during the course of mineral growth is an encouraging scaffold design result for tissue regeneration. Materials with greater compressive moduli tend to have a heightened ability to withstand the forces of cellular contraction during tissue development and avoid scaffold contraction. ${ }^{6}$ This translates into a material that exhibits shape memory, so that the regenerated tissue will form in the shape of the preexisting synthetic scaffold. Previous studies indicate that some poly $(\alpha-$ hydroxy acid) constructs are insufficient for resistance of cellular contractile forces during tissue development in vitro ${ }^{6}$ and in vivo ${ }^{25}$ and call for novel techniques for scaffold reinforcement. Notably, the com- pressive moduli of the scaffolds in this study can be increased by incubation in SBF without notable effects on total scaffold porosity or pore size.

The approach developed in this study to mineralize porous, resorbable, biocompatible polymer scaffolds offers several design advantages in the context of tissue engineering. The continuous BLM layer is expected to be osteoconductive and resorbable in vivo, unlike stoichiometric hydroxyapatite, which is too stable to be adequately resorbed. ${ }^{40}$ High scaffold porosity is maintained, which allows for cell migration and vascular infiltration. The mechanical integrity of the scaffold is enhanced by the presence of the mineral film, offering the possibility of engineering tissues in predefined shapes without scaffold contraction during tissue development.

\section{CONCLUSIONS}

A simple hydrolysis reaction on the surface of a PLG scaffold was shown to be an adequate functionalization technique for mineral formation. Mineral formation was accomplished via a single step, room temperature process. A continuous carbonated apatite mineral was formed within pores upon incubation in an SBF for 16 days. Such a layer could be of great importance in the field of bone tissue engineering, providing the advantages of increased osteoconductivity and enhanced mechanical properties. Also, because this is a one step, room temperature process, it is an efficient and relatively inexpensive mineral growth process.

\section{References}

1. Poss R, editor. Orthopaedic knowledge update. Volume 3. Chicago, IL: American Academy of Orthopaedic Surgeons; 1990.

2. Langer R, Vacanti JP. Tissue engineering. Science 1993;260:920926.

3. Crane GM, Ishaug SL, Mikos AG. Bone tissue engineering. Nat Med 1995;1:1322-1324.

4. Putnam AJ, Mooney DJ. Tissue engineering using synthetic extracellular matrices. Nat Med 1996;2:824-826.

5. Minabe M. A critical review of the biologic rationale for guided tissue regeneration. J Periodontol 1991;62:171-179.

6. Kim BS, Mooney DJ. Engineering smooth muscle tissue with a predefined structure. J Biomed Mater Res 1998;41:322-332.

7. Pomahac B, Svensjo T, Yao F, Brown H, Eriksson E. Tissue engineering of skin. Crit Rev Oral Biol Med 1998;9:333-344.

8. Bunker BC, Rieke PC, Tarasevich BJ, Campbell AA, Fryxell GE, Graff GL, Song L, Liu J, Virden W, McVay GL. Ceramic thinfilm formation on functionalized interfaces through biomimetic processing. Science 1994;264:48-55.

9. Vallet-Regi M, Romero AM, Ragel CV, LeGeros RZ. XRD, SEM-EDS, and FTIR studies of in vitro growth of an apatite-like layer on sol-gel glasses. J Biomed Mater Res 1998;44:416-421.

10. Li P, Nakanishi K, Kokubo T, deGroot K. Induction and mor- 
phology of hydroxyapatite, precipitated from metastable simulated body fluids on sol-gel prepared silica. Biomaterials 1993; 14:963-968.

11. Abe Y, Kokubo T, Yamamuro T. Apatite coating on ceramics, metals, and polymers utilizing a biological process. J Mater Sci Mater Med 1990;1:233-238.

12. Campbell AA, Fryxell GE, Linehan JC, Graff GL. Surfaceinduced mineralization: a new method for producing calcium phosphate coatings. J Biomed Mater Res 1996;32:111-118.

13. Wen HB, deWijn JR, Cui FZ, deGroot K. Preparation of calcium phosphate coatings on titanium implant materials by simple chemistry. J Biomed Mater Res 1997;41:227-236.

14. Tretinnikov ON, Kato K, Ikada Y. In vitro hydroxyapatite deposition onto a film surface grafted with organophosphate polymer. J Biomed Mater Res 1994;28;1365-1373.

15. Tanahashi M, Yao T, Kokubo T, Minoda M, Miyamoto T, Nakamura T, Yamamuro T. Apatite coated on organic polymers by biomimetic process: improvement in adhesion to substrate by glow-discharge treatment. J Biomed Mater Res 1995;29:339_ 347.

16. Hench LL. Bioceramics: from concept to clinic. J Am Ceram Soc 1991;74:1487-1510.

17. Kokubo T. Recent progress in glass based materials for biomedical applications. J Ceram Soc Jpn 1991;99:965-973.

18. Kitsugi T, Yamamuro T, Nakamura T, Higashi S, Kakutani $Y$, Hyakuna K, Ito S, Kokubo T, Takagi M, Shibuya T. Bone bonding behaviour of three kinds of apatite-containing glassceramics. J Biomed Mater Res 1986;20:1295-1307.

19. LeGeros RZ, Daculsi G. In vivo transformation of biphasic calcium phosphate ceramics: ultrastructural and physicochemical characterizations. In: Yamamuro T, Hench LL, Wilson J, editors. Handbook of bioactive ceramics. Boca Raton, FL: CRC Press; 1990. p 17-28.

20. Hench LL, Wilson J. Surface-active biomaterials. Science 1984; 226:630-636.

21. Oyane A, Nakanishi K, Kim H, Miyaji F, Kokubo T, Soga N, Nakamura T. Sol-gel modification of silicone to induce apatiteforming ability. Biomaterials 1999;20:79-84.

22. Miyaji F, Kim HM, Handa S, Kokubo T, Nakamura T. Bonelike apatite coating on organic polymers: novel nucleation process using sodium silicate solution. Biomaterials 1999;20:913-919.

23. Varma HK, Yokogawa Y, Espinosa FF, Kawamoto Y, Nishizawa K, Nagata F, Kameyama T. Porous calcium phosphate coating over phosphorylated chitosan film by a biomimetic method. Biomaterials 1999;20:879-884.

24. Taguchi T, Shiraogawa M, Kishida A, Akashi M. A study on hydroxyapatite formation on/in the hydroxyl groups-bearing nonionic hydrogel. J Biomater Sci Polym Ed 1999;10:19-32.

25. Kikuchi M, Tanaka J, Koyama Y, Takakuda K. Cell culture test of TCP/CPLA composite. J Biomed Mater Res Appl Biomater 1999;48:108-110.

26. Mikos AG, Thorsen AJ, Czerwonka LA, Bao Y, Langer R, Winslow DN, Vacanti JP. Preparation and characterization of poly(L-lactic acid) foams. Polymer 1994;35:1068-1077.

27. Weast RC, Astle MJ. CRC Handbook of chemistry and physics. 63rd ed. Boca Raton, FL: CRC Press; 1983. p B228.

28. Heinonen JK, Lahti RJ. A new and convenient colorimetric determination of inorganic orthophosphate and its application to the assay of inorganic pyrophosphatase. Anal Biochem 1991; 113:313-317.

29. Rehman I, Bonfield W. Characterization of hydroxyapatite and carbonated apatite by photo acoustic FTIR spectroscopy. J Mater Sci Mater Med 1997;8:1-4.

30. Silverstein RM, Bassler GC, Merrill TC. Spectrometric identification of organic compounds. 5th ed. New York: Wiley; 1991. p 91-165.

31. Gao JM, Niklason L, Langer R. Surface hydrolysis of poly(glycolic acid) meshes increases the seeding density of vascular smooth muscle cells. J Biomed Mater Res 1998;42:417-424.

32. Lee $\mathrm{S}$, Veis A. Cooperativity in calcium ion binding to repetitive, carboxylate-serylphosphate polypeptides and the relationship of this property to dentin mineralization. J Peptide Protein Res 1980;16:231-240.

33. Weiner SJ. Aspartic acid-rich proteins: major components of the soluble organic matrix of mollusk shells. Calcif Tissue Int 1979;29:163-167.

34. Lowenstam HA, Weiner S. On biomineralization. Oxford, U.K.: Oxford University Press; 1989.

35. Kim HM, Kishimoto K, Miyaji F, Kokubo T, Yao T, Suetsugu Y, Tanaka J, Nakamura T. Composition and structure of the apatite formed on PET substrates in SBF modified with various ionic activity products. J Biomed Mater Res 1999;46:228-235.

36. Zhang R, Ma PX. Porous poly(L-lactic acid)/apatite composites created by biomimetic process. J Biomed Mater Res 1999; 45:285-293.

37. Wake MC, Gupta PK, Mikos AG. Fabrication of pliable biodegradable polymer foams to engineer soft tissues. Cell Transplant 1996;5:465-473.

38. Thompson RC, Yaszemski MJ, Powers JM, Mikos AG. Fabrication of biodegradable polymer scaffolds to engineer trabecular bone. J Biomater Sci Polym Ed 1995;7:23-38.

39. Shea LD, Wang D, Franceschi RT, Mooney DJ. Bone formation from pre-osteoblasts on 3-d scaffolds, submitted.

40. Frayssinet P, Trouillet JL, Rouquet N, Azimus E, Autefage A. Osseointegration of macroporous calcium phosphate ceramics having a different chemical composition. Biomaterials 1993;14: 423-429. 